\title{
Desenvolvimento e Propriedades Psicométricas da Escala de Atitude em Relação à Ciência
}

\author{
Felipe Carvalho Novaes ${ }^{1}$ \\ Bheatrix Bienemann ${ }^{1}$ \\ Fernanda de Oliveira Paveltchuke ${ }^{2}$ \\ Pedro Henrique Teodoro Siqueira ${ }^{2}$ \\ Bruno Figueiredo Damásio ${ }^{2}$ \\ ${ }^{1}$ Pontifícia Universidade Católica do Rio de Janeiro, Rio de Janeiro, RJ \\ ${ }^{2}$ Universidade Federal do Rio de Janeiro, Rio de Janeiro, RJ
}

\begin{abstract}
Resumo
Este estudo apresenta o desenvolvimento e evidências de validade da Escala de Atitude em Relação à Ciência (EARC). Participaram 697 indivíduos (53,5\% homens), com idades entre 18 e 75 anos $(M=27,51$ e $D P=9,8)$ de diversos estados brasileiros. Análise fatorial exploratória (AFE) e análises fatoriais confirmatórias (AFC) demonstraram que a EARC apresenta estrutura de dois fatores (Iniciativa Pessoal e Crenças \& Afetos). As comparações da EARC com variáveis sociodemográficas revelaram que homens têm atitude em relação à ciência (ARC) mais favorável que as mulheres, não religiosos apresentaram maior ARC que religiosos, estudantes de Ensino Superior em instituições públicas apresentaram maior ARC que os de instituições privadas, participantes de exatas apresentaram maior ARC que os de cursos de humanas e a variável Necessidade de Conhecimento se correlacionou positivamente com ARC. A EARC revelou adequação confiável e válida para mensurar atitude em relação à ciência em amostras brasileiras.
\end{abstract}

Palavras-chave: ciência, atitude, escala, mensuração

Development and Psychometric Properties of the Attitude Toward Science Scale

\begin{abstract}
This study presents the development and evidence of validity of the Attitude Toward Science Scale (ATSS). Participants included 697 individuals $(53.5 \%$ men), aged between 18 and 75 years $(M=27.51, S D=9.8)$ in several Brazilian states. Exploratory factorial analysis (EFA) and confirmatory factor analysis (CFA) showed that ATSS has a two-factor structure (Personal Initiative and Beliefs \& Affects). ATSS comparisons with sociodemographic variables revealed that men have a more favorable attitude towards science (ATS) than women, non-religious individuals show more favorable ATS than religious ones, students of public universities have more favorable ATS than those of private universities, students of hard sciences show more favorable ATS than those of humanities and a positive correlation between 'need for knowledge' and ATS. ATSS shows reliable and valid adequacy for measuring attitude towards science in Brazilian samples.
\end{abstract}

Key-words: science; attitude; scale; measurement

\section{Desenvolvimento y Propiedades Psicométricas de la Escala de Actitud en Relación con la Ciencia}

\section{Resumen}

Este estudio presenta desarrollo y evidencias de validez de la Escala de Actitud con Relación a la Ciencia (EARC). Participaron 697 individuos (53,5\% hombres) con edades comprendidas entre los 18 y 75 años $(M=27,51, S D=9,8)$ de diversos estados brasileños. Análisis Factorial Exploratorio (AFE) y Análisis Factorial Confirmatorio (AFC) demostraron que la EARC presenta estructura de dos factores (Iniciativa Personal y Creencias \& Afectos). Comparaciones de la EARC con variables sociodemográficas revelaron que los hombres tienen actitudes más favorables que las mujeres con relación a la ciencia (ARC); las personas que no practican ninguna religión presentaron mayor ARC que los que practican, estudiantes de Universidades públicas presentaron mayor ARC que los de Universidades privadas, estudiantes de ciencias exactas presentaron mayor ARC que los de cursos de humanidades, encontrándose una correlación positiva entre la variable "necesidad de conocimiento" y ARC. La escala EARC reveló una adecuación confiable y válida para medir la actitud hacia la ciencia en muestras brasileñas.

Palabras clave: ciencia; actitud; escala; medición

Conhecimento científico pode ser definido como um tipo de conhecimento que fora submetido ao método científico, isto é, cujas hipóteses foram testadas (Popper, 1959/2004). Esse método de aquisição de conhecimento tem extrema importância na prevenção contra vieses nas pesquisas (Lilienfeld, 2010) tendo trazido, ao longo da história, diversos benefícios à humanidade devido ao avanço tecnológico propiciado por meio do conhecimento adquirido cientificamente (Sagan, 2006).

Atualmente, porém, tem crescido a oposição dentro das ciências humanas contra a concepção moderna 
de ciência, particularmente, contra a ideia de objetividade (Castañon, 2009a) e racionalidade científica (Bland, 2014). Esse movimento fora bastante impulsionado pela influência de correntes filosóficas com visões epistemológicas pós-modernistas nas ciências humanas (Castañon, 2009a), cujos preceitos se mostram, muitas vezes, incompatíveis com os pressupostos ontológicos necessários para a prática científica (Bienemann \& Damásio, 2017; Castañon, 2009b). Outro ponto importante a se destacar é o recente desinteresse e analfabetismo científico por parte da sociedade, culminando em sentimentos "anticiência" e ascensão de crenças pseudocientíficas entre os cidadãos e seus governantes, expressas, por exemplo, em movimentos contra vacinação, negação das mudanças climáticas, profecias de fim de mundo, entre outros (Lobato \& Zimmerman, 2018).

A atitude em relação à ciência (ARC) pode ser definida como um interesse e engajamento em disciplinas científicas e busca por carreiras científicas por parte da população (Osborne, Simon, \& Collins, 2003). Evidências relacionam ARC à qualidade do ensino de ciência e do pensamento científico nas escolas (van Aalderen-Smeets \& van der Molen, 2015), sendo que o pensamento científico pode ser definido como um modo de pensar que requer habilidades e perícia para que uma pessoa tenha suas crenças pessoais alinhadas com evidências para essas crenças (Kuhn, 2005).

No presente estudo foi utilizada a definição de ARC como sendo a composição de crenças, afetos e comportamentos de um indivíduo em relação à ciência (Greenwald, Brock, \& Ostrom, 2013), conforme o modelo clássico da teoria das atitudes (Rodrigues, Assmar, \& Jabonski, 2001). Isto é, a atitude em relação à ciência seria estruturada pelas crenças dos indivíduos em relação à ciência, pelos afetos positivos e negativos em relação à ciência, e pelos seus comportamentos em relação à ciência (i.e., o quanto lêem sobre ciência, o quanto pesquisam sobre ciência etc.).

Estudos demonstram que os níveis de ARC parecem sofrer influência de algumas variáveis, tais como gênero (Smith, Pasero, \& McKenna, 2014), nível educacional (Antonioli, 2012; Perry et al., 2012) e necessidade de conhecimento (Feist, 2012). Alguns estudos obtiveram resultados que indicam uma ARC mais positiva por parte de crianças do sexo masculino, que também parecem demonstrar maior confiança no método científico e em seus resultados do que as de sexo feminino (Smith et al., 2014; Wang \& Berlin, 2010; Weinburgh, 1995). Uma provável responsável para esse dado é a maior influência social recebida pelos homens, desde a infância, a seguirem carreiras científicas e a gostar e se envolver com ciência mais do que as mulheres (Reuben, Sapienza, \& Zingales, 2014; Smith et al., 2014).

O nível educacional das pessoas também é um provável fator que impacta na $\mathrm{ARC}$, uma vez que o ensino de ciência nas escolas está amplamente relacionado com a construção dessas atitudes (Antonioli, 2012; van Aalderen-Smeets \& van der Molen, 2015). Além disso, a necessidade de conhecimento, i.e., o nível de importância que as pessoas atribuem à aquisição de conhecimento em suas vidas (Schnell \& Becker, 2007), também é uma provável influenciadora nas atitudes. Uma vez que a ciência está relacionada com aquisição de conhecimento confiável e com racionalidade (Sagan, 2006), e que os cientistas tendem a ter níveis altos de necessidade de conhecimento (Feist, 2012), espera-se que esse construto se correlacione com ARC.

\section{Mensurando Atitude em Relação à Ciência (ARC)}

$\mathrm{O}$ interesse em investigar a ARC tem motivado a criação de instrumentos para mensurar esse construto. Apesar de existirem escalas que mensuram ARC, é possível observar alguns problemas psicométricos em muitas delas. Blalock et al. (2008), em uma revisão, observou que existem 150 estudos sobre ARC, atitudes científicas, natureza da ciência, interesse por carreiras científicas e outros. Ao todo, eram 66 instrumentos criados para mensurar esses construtos, sendo 20 deles destinados a mensurar especificamente ARC. No entanto, o autor constatou que muitos instrumentos não foram replicados por outros estudos e/ou possuem evidências de validade insatisfatórias (Blalock et al., 2008). Mais especificamente, cerca de $40 \%$ dos estudos que buscavam mensurar ARC não tinham algum componente fundamental de evidência psicométrica, como, por exemplo, consistência interna satisfatória (Blalock et al., 2008). Ademais, alguns instrumentos têm uso específico ao ambiente escolar infantil, o que limita sua aplicabilidade, como é o caso da Attitudes Toward Science School Assessment (Germann, 1988), a escala para mensurar ARC que parece ter sido a mais utilizada pelos estudos ao redor do mundo (Blalock et al., 2008). Esse e outros instrumentos costumam ser voltados para estudantes e têm foco no ensino científico (Krapp \& Prenzel, 2011). Uma recente revisão metodológica sobre ARC em europeus também destacou a fragilidade dos instrumentos de mensuração existentes e a necessidade de se criar novos (Pardo \& Calvo, 2016). Até onde se sabe, não houve tentativas de desenvolvimento 
e validação de instrumentos destinados a medir ARC na população em geral desde então.

No Brasil, investigações sobre ARC são ainda escassas. Com o objetivo de levantar os possíveis instrumentos já desenvolvidos na literatura nacional que buscassem avaliar ARC, foi realizada uma pesquisa não sistemática utilizando o mecanismo de busca Google Scholar, o Portal de Periódicos da CAPES, além das bases de dados PePSIC e SciELO. Foram utilizados os descritores "ciência", "instrumentos", "questionários", "escalas", "atitude", "atitude relacionada à ciência" e "atitude em relação à ciência". Foram encontrados apenas três estudos avaliando ARC. Cunha (2008) verificou concepções de docentes sobre desenvolvimento científico e tecnológico e as relações desse desenvolvimento com a sociedade, utilizando, para isso, uma escala para avaliar as atitudes frente às interações entre ciência, sociedade e tecnologia. Antonioli (2012) tentou compreender como estudantes se posicionam em relação a questões que envolvem o cotidiano, a ciência e os cientistas. Para tanto, utilizou o Questionário de Atitudes sobre Ciência, Tecnologia e Sociedade (COCTS). Já Constantino (2004) buscou analisar o efeito do ensino de ciência e tecnologia em idades precoces. Esses exemplos de estudos apontam algumas limitações nas escalas de ARC, tais como elas serem voltadas para grupos muito específicos - geralmente alunos de escolas e que focam não só em ciência como em tecnologia científica. Uma revisão de 12 anos de pesquisa, realizada por Potvin e Hasni (2014) também constatou que nenhum dos instrumentos encontrados para mensurar ARC havia utilizado amostras sul-americanas.

Sendo assim, dada a importância social da ciência (Sagan, 2006) e os problemas existentes nos instrumentos destinados a mensurar ARC que já estão publicados, faz-se necessário uma escala de ARC com maior restrição temática, isto ém que busque avaliar como as pessoas se relacionam à ciência especificamente, e não aos benefícios proporcionados por ela, e com maior abrangência e especificidade amostral. Desse modo, este estudo buscou desenvolver a Escala de Atitude em Relação à Ciência (EARC), um instrumento destinado a mensurar ARC na população geral, assim como verificar possíveis relações existentes entre ARC e outros construtos.

\section{Hipóteses}

Com base no modelo atitudinal clássico (Rodrigues et al., 2001) e nas relações da ARC com outros construtos (Antonioli, 2012; Perry et al., 2012; Feist, 2012; Smith et al., 2014), as seguintes hipóteses foram elaboradas: 1) A EARC será composta por três fatores - crenças, afetos e comportamentos; 2) Nível de escolaridade e ARC se correlacionarão positivamente; 3) Homens terão ARC mais favorável do que mulheres; e 4) Necessidade de conhecimento e ARC se correlacionarão positivamente.

\section{Método}

\section{Participantes}

Participou desta pesquisa uma amostra de 697 pessoas (53,5\% homens). O perfil etário da amostra variou de 18 aos 75 anos $(M=27,51$ e $D P=9,8)$. A maioria dos participantes indicou ter Ensino Superior Incompleto $(n=262)$, seguido pelos que possuem pós-graduação $(n=161)$, até Ensino Médio Completo $(n=$ 144) e Ensino Superior Completo $(n=130)$. Dos participantes ainda cursando o Ensino Superior $(n=262)$, 178 estão em IES (instituições de Ensino Superior) públicas e 84 em IES privadas. Dos alunos cursando especificamente a graduação, $53,1 \%$ estão em cursos da área de Humanas, Administração e Artes; $24,7 \%$ nas áreas Exatas e 22,3\% em Biológicas e Química.

\section{Instrumentos}

Escala de Atitude em Relação à Ciência (EARC). Trata-se de um instrumento que busca mensurar atitude em relação à ciência na população geral.

\section{Procedimentos de Construção da EARC}

Inicialmente, foram elaborados itens que teoricamente acoplassem aspectos emocionais (como se sentem), cognitivos (o que pensam) e comportamentais (como se comportam) sobre a ciência em geral. Os itens foram avaliados por três juízes externos, experts na área de construção de testes, especialistas em psicometria e construção de instrumentos, que avaliaram a validade de conteúdo dos itens. Após ajustes menores baseados nas sugestões dos juízes, os itens passaram por um estudo piloto, onde uma amostra da população à qual a escala se destina avaliou o instrumento $(N=$ 14). Após esse estudo piloto, a EARC foi considerada pronta para aplicação e posterior avaliação psicométrica (Borsa, Damásio \& Bandeira, 2012). O instrumento é respondido em uma escala tipo Likert de cinco pontos, variando de 1 (discordo totalmente) a 5 (concordo totalmente).

\section{Instrumentos Adicionais}

Questionário Sociodemográfico. Esse questionário teve por objetivo levantar informações 
Tabela 1.

Características Sociodemográficas da Amostra

\begin{tabular}{|c|c|c|c|c|c|c|}
\hline \multirow{3}{*}{ Idade } & \multicolumn{2}{|c|}{ Total } & \multicolumn{2}{|c|}{ Homens } & \multicolumn{2}{|c|}{ Mulheres } \\
\hline & $M$ & $D P$ & $M$ & $D P$ & $M$ & $D P$ \\
\hline & 24,44 & 9,78 & 26,67 & 8,88 & 28,33 & 10,67 \\
\hline Idade em categorias & $N$ & $\%$ & $n$ & $\%$ & $n$ & $\%$ \\
\hline $18-25$ anos & 384 & 55,1 & 212 & 56,8 & 172 & 53,1 \\
\hline 26-35 anos & 204 & 29,3 & 116 & 31,1 & 88 & 27,2 \\
\hline $36-55$ anos & 75 & 10,8 & 33 & 8,8 & 42 & 13 \\
\hline 56-75 anos & 34 & 4,9 & 12 & 3,2 & 22 & 6,8 \\
\hline \multicolumn{7}{|l|}{ Região do Brasil } \\
\hline Sudeste & 460 & 66 & 241 & 64,6 & 219 & 67,6 \\
\hline Sul & 103 & 14,8 & 57 & 15,3 & 46 & 14,2 \\
\hline Nordeste & 63 & 9 & 31 & 8,3 & 32 & 9,9 \\
\hline Centro-Oeste & 41 & 5,9 & 27 & 7,2 & 14 & 4,3 \\
\hline Norte & 30 & 4,3 & 17 & 4,6 & 13 & 4 \\
\hline \multicolumn{7}{|l|}{ Área de formação } \\
\hline Humanas, Adm. e Artes & 155 & 22,2 & 59 & 39,6 & 96 & 67,1 \\
\hline Exatas & 72 & 10,3 & 56 & 37,6 & 16 & 11,2 \\
\hline Biológicas e Química & 65 & 9,3 & 34 & 22,8 & 31 & 21,7 \\
\hline \multicolumn{7}{|l|}{ Local de estudo } \\
\hline Sudeste & 30 & 79,9 & 16 & 84,2 & 14 & 70 \\
\hline Sul & 6 & 15,4 & 3 & 15,8 & 3 & 15 \\
\hline Nordeste & 2 & 5,1 & 0 & 0 & 2 & 10 \\
\hline Centro-Oeste & 1 & 2,6 & 0 & 0 & 1 & 5 \\
\hline \multicolumn{7}{|l|}{ Crença Religiosa } \\
\hline Sim & 353 & 50,6 & 155 & 41,6 & 198 & 61,1 \\
\hline Não & 344 & 49,4 & 218 & 58,4 & 126 & 38,9 \\
\hline \multicolumn{7}{|l|}{ Estado Civil } \\
\hline Solteiro & 484 & 69,4 & 275 & 73,3 & 209 & 64,5 \\
\hline Casado & 105 & 15,1 & 53 & 14,2 & 52 & 16 \\
\hline União Estável & 64 & 9,2 & 30 & 8 & 34 & 10,5 \\
\hline Noivo & 21 & 3 & 8 & 2,1 & 13 & 4 \\
\hline Divorciado & 21 & 3 & 7 & 1,9 & 14 & 4,3 \\
\hline Viúvo & 2 & 0,3 & 0 & 0 & 2 & 0,6 \\
\hline União Civil & 0 & 0 & 0 & 0 & 0 & 0 \\
\hline
\end{tabular}

Nota. $M=$ média; $D P=$ desvio padrão; $N=$ número total de participantes; $n=$ número amostral por grupo. 
sociodemográficas, tais como sexo, idade, grau de instrução, local de residência, tipo de instituição de ensino superior a qual o participante estuda/estudou (pública ou privada), local de formação etc.

Subescala de Necessidade de Conhecimento (SNC; Schnell \& Becker, 2007; adaptado por Damásio, Koller, \& Schnell, 2013). A SNC é uma subescala do Souces of Meaning and Meaning in Life Questionnaire (SoMe). É composta por cinco itens que avaliam, em uma escala de seis pontos $(0=$ discordo totalmente; $5=$ concordo totalmente) a importância ou necessidade de conhecimento na vida dos sujeitos. Quanto maior os escores, maior é a importância que o indivíduo dá para a necessidade de conhecimento. No presente estudo, a consistência interna da escala (alfa de Cronbach) foi de $\alpha=0,81$.

\section{Procedimentos de Coleta de Dados}

A coleta de dados foi realizada virtualmente, por meio de uma plataforma on-line. O link para participação da pesquisa foi divulgado exaustivamente em redes sociais e por listas de e-mails. O questionário da pesquisa só tinha início conforme o participante concordasse e aceitasse o Termo de Consentimento Livre e Esclarecido (TCLE), presente logo na primeira página da pesquisa. A privacidade dos participantes, bem como o sigilo e confidencialidade dos dados foram garantidas e eles foram informados de que poderiam abandonar a pesquisa a qualquer momento, sem nenhum tipo de prejuízo ou consequência negativa. O estudo teve aprovação mediante comitê de ética da Universidade Federal do Rio de Janeiro, tendo por base as diretrizes da Resolução 510/2016, que regulamenta a pesquisa com seres humanas nas ciências sociais e humanas (Número de Protocolo CAAE 38318314.2.0000.5582). Os critérios de inclusão do estudo eram ser brasileiro, residente atual no Brasil e maior de idade.

\section{Procedimentos de Análise de Dados}

\section{Estrutura Fatorial da EARC}

Inicialmente a amostra foi dividida, de maneira aleatória, em aproximadamente duas partes iguais. Com a primeira parte da amostra $(n=346)$, foi avaliado, por meio do software Factor v.9.2 (Lorenzo-Seva \& Ferrando, 2006), o melhor número de fatores a ser retido para a EARC. Nesse processo, foram utilizados dois critérios distintos, sendo: 1) Análise paralela (Horn, 1965) e 2) Método Hull (Lorenzo-Seva, Timmerman, \& Kiers, 2011). A seguir, avaliou-se estrutura fatorial da EARC por meio da técnica de Análise Fatorial Exploratória
(AFE; Muthén \& Muthén, 2012), utilizando o método de estimação Weighted Least Squares Mean-and-variance Adjusted (WLSMV; Muthén \& Muthén, 2012), adequado para variáveis ordinais com desvios de normalidade.

Com base nos resultados da EFA, procedimentos de refinamento da escala foram implementados para torná-la mais parcimoniosa. Assim, optou-se por excluir os itens que tivessem carga cruzada acima de 0,30 em mais de um fator ou apresentassem cargas fatoriais abaixo de 0,40 (Pituch \& Stevens, 2015). Após o refinamento, buscou-se avaliar a sua adequação da nova estrutura da EARC. Para isso, foram realizadas análises fatoriais confirmatórias (AFCs) com a segunda parte da amostra $(n=345)$, utilizando o mesmo método de estimação da etapa anterior (WLSMV).

Os índices de ajuste utilizados na AFE e na AFC foram o Root Mean Square error of Approximation (RMSEA), Comparative Fit Index (CFI), Tucker-Lewis Index (TLI). De acordo com os critérios utilizados (Brown, 2006), valores de RMSEA ( $90 \%$ IC) não devem ser superiores a 0,10 , e valores de CFI e TLI devem ser maiores que 0,90 , preferencialmente acima de 0,95 . A consistência interna da versão final da escala foi avaliada por meio da fidedignidade composta.

\section{Associações Sociodemográficas com os niveis de ARC}

Após a delimitação da estrutura fatorial da escala, buscou-se investigar associações nos níveis de ARC frente a algumas características sociodemográficas. Inicialmente, foram realizadas correlações de Pearson entre a idade e os níveis de EARC. Posteriormente, foram realizados testes $t$ de Student para comparar os escores da EARC em relação ao sexo, religiosidade (presença ou ausência) e entre estudantes do ensino superior de universidades públicas e privadas. Ainda, buscou-se investigar, por meio de uma análise de variância (ANOVA), se os níveis de ARC variavam entre pessoas com diferentes níveis de escolaridade $(\mathrm{EMc}=$ Até Ensino Médio completo; ESc = até Ensino Superior completo; $\mathrm{Ec}=$ até Especialização Completa; $\mathrm{Mc}=$ até Mestrado completo; Dc = até Doutorado completo) e entre universitários de diferentes áreas do conhecimento (Área 1 = Humanas, incluindo administração e artes; Área 2 = Exatas; e Área 3 = Biológicas, incluindo química). Para todas as análises, foram implementados procedimentos de re-amostragem (bootstrapping; 1000 re-amostragens, com intervalo de confiança 99\%), com vistas a apresentar maior confiabilidade aos resultados, corrigir possíveis desvio de normalidade da amostra, e apresentar intervalo de confiança de $99 \%$ para as 
diferenças de média (Haukoos \& Lewis, 2005). O tamanho de efeito da ANOVA geral foi calculado utilizando o eta-squared $\left(\eta^{2}\right)$. O tamanho de efeito para as comparações par-a-par (post-hoc) foi calculado por meio do d de Cohen (Cohen, 1988).

\section{Resultados}

Inicialmente, foram gerados 60 itens no processo de elaboração. Ao final dos procedimentos de criação dos itens, itens repetitivos, semanticamente semelhantes ou gramaticalmente complexos foram eliminados. No fim do procedimento, restaram 50 itens na EARC. Depois, a escala foi avaliada por juízes e foi submetida a um estudo piloto $(N=14)$, no qual nenhum item foi eliminado, sendo feitos apenas ajustes gramaticais, semânticos ou idiomáticos mantendo o total de 50 itens na escala.

Buscou-se investigar a dimensionalidade da EARC utilizando, enquanto técnicas de retenção fatorial, a análise paralela (Timmerman \& Lorenzo-Seva, 2011) e o método Hull (Lorenzo-Seva et al., 2011). As diferentes técnicas apontaram para dois e um fator, respectivamente. Considerando esses resultados, foram executadas duas AFE, com o objetivo de investigar a estrutura teórica da EARC composta por um e dois fatores (Ver Tabela 2).

Conforme pode ser visto na Tabela 2, na estrutura unifatorial, todos os itens carregam adequadamente, a exceção dos itens 8 e 27, que apresentaram cargas fatoriais abaixo de 0,40 . No que se refere à estrutura de dois fatores, é possível observar que os itens $2,11,12$, 27, 39 e 47 não carregaram adequadamente em nenhum fator, enquanto os itens 8 e 19 apresentaram carga cruzada, com carga fatorial acima de 0,30 nos dois fatores. Ainda, os índices de ajuste do modelo de dois fatores apresentam-se mais adequados do que quando comparado com o modelo unifatorial [Unifatorial, $\chi^{2}=$ 4168.601, $g l=1175$; RMSEA $(90 \%$ IC $)=0,086(0,083$ $-0,089)$; CFI $=0,890$; TLI $=0,886$; Dois fatores: $\chi^{2}=$ $2722,221, g l=1126 ;$ RMSEA $(90 \%$ IC) $=0,064(0,061$ $-0,067)$; CFI $=0,942$; TLI $=0,936]$.

Ao avaliar teoricamente os dois fatores, pode-se perceber que o primeiro fator se refere a comportamentos e aspirações pessoais relacionados à ciência, sendo denominado "Iniciativa Pessoal" (IP); enquanto o segundo fator se refere a cognições e afetos em relação à ciência de maneira geral, sem ter relação com a pessoa propriamente dita, sendo denominado "Crenças e afetos" (CA). Considerando a interpretabilidade e adequação teórica da estrutura de dois fatores, bem como seus adequados índices de ajuste, optou-se por acatar essa estrutura em detrimento da unifatorial. Assim, os processos de refinamento da EARC foram implementados de acordo com os resultados da estrutura de dois fatores.

Conforme mencionado anteriormente, com vistas a tornar a escala mais parcimoniosa, no processo de refinamento, foram excluídos os itens que apresentaram baixa carga fatorial $(<0,40)$ ou carga cruzada (carga fatorial $>$ de $0,30 \mathrm{em}$ mais de um fator). Assim, foram excluídos os itens 2, 11, 12, 19, 27, 39, 46 e 47 (Ver Tabela 2), de modo que a EARC ficou composta por 42 itens.

Com o objetivo de investigar a adequação da escala refinada, foram executadas, com a segunda parte da amostra, duas análises fatoriais confirmatórias (AFC). A primeira AFC foi implementada em uma estrutura unifatorial; a segunda AFC foi implementada numa estrutura de dois fatores, sem a presença de cargas cruzadas. O critério de composição dos itens em cada um dos fatores da EARC deu-se por base nas cargas fatoriais dos itens apresentada na tabela 2 (itens com carga acima de 0,40 foram considerados como "pertencentes" a determinado fator). Os índices de ajuste do modelo unifatorial foram: RMSEA 0,096 (0,093-0,099); $\mathrm{CFI}=0,88 ;$ TLI $=0,873$. Para o modelo bidimensional foram: RMSEA 0,064 (0,060-0,067); CFI = 0,95; TLI $=0,94$.

Conforme pode ser visto, a estrutura de dois fatores refinada apresentou índices de ajuste adequados e superiores aos do modelo unifatorial, o que atesta, novamente, que a EARC é mais bem concebida como um instrumento bidimensional. A confiabilidade dos fatores, medido por meio da fidedignidade composta (FC), apresentou, também, resultados adequados: Fator $\mathrm{IP}, \mathrm{FC}=0,96$; Fator $\mathrm{CA}, \mathrm{FC}=0,94$.

\section{Atitude em Relação à Ciência e Relações com Variáveis Sociodemográficas}

Uma ANOVA one-way foi realizada com o objetivo de comparar os níveis de ARC (Fator IP e CA) para pessoas com diferentes níveis de escolaridade $(\mathrm{EMc}=$ Até Ensino Médio completo; ESc = até Ensino Superior completo; $\mathrm{Ec}=$ até Especialização Completa; $\mathrm{Mc}=$ até Mestrado completo; Dc = até Doutorado completo). $O$ fator IP apresentou diferenças estatisticamente significativas entre diferentes níveis de escolaridade $[F(4$, 686) $\left.=6.66 ; p=0,000 ; \eta^{2}=0,037\right]$. Já o fator CA não apresentou diferenças estatisticamente significativas 
Tabela 2

Estruturas Fatoriais da EARC (Uni e Multidimensional)

\begin{tabular}{|c|c|c|c|c|c|c|c|}
\hline \multirow{2}{*}{ Itens } & \multirow{2}{*}{$\begin{array}{l}\text { Estrutura } \\
\text { unifatorial }\end{array}$} & \multicolumn{2}{|c|}{ Estrutura de dois fatores } & \multirow{2}{*}{ Itens } & \multirow{2}{*}{$\begin{array}{l}\text { Estrutura } \\
\text { unifatorial }\end{array}$} & \multicolumn{2}{|c|}{ Estrutura de dois fatores } \\
\hline & & Fator 1 & Fator 2 & & & Fator 1 & Fator 2 \\
\hline Item 1 & $-0,637$ & 0,018 & $0,652^{*}$ & Item 26 & 0,430 & 0,078 & $-0,527 *$ \\
\hline Item 2 & $-0,640$ & 0,284 & 0,398 & Item 27 & 0,173 & 0,019 & 0,200 \\
\hline Item 3 & $-0,630$ & $-0,259$ & $0,912^{*}$ & Item 28 & $-0,617$ & $-0,169$ & $0,810^{*}$ \\
\hline Item 4 & $-0,749$ & 0,159 & $0,663^{*}$ & Item 29 & 0,526 & $-0,777^{*}$ & 0,195 \\
\hline Item 5 & 0,543 & $-0,621 *$ & $-0,028$ & Item 30 & $-0,799$ & $0,908^{*}$ & $-0,035$ \\
\hline Item 6 & $-0,633$ & $0,941 *$ & $-0,241$ & Item 31 & $-0,564$ & $0,569^{*}$ & 0,044 \\
\hline Item 7 & 0,534 & 0,173 & $-0,727 *$ & Item 32 & $-0,454$ & $-0,079$ & $0,553^{*}$ \\
\hline Item 8 & 0,349 & 0,058 & $-0,422 *$ & Item 33 & $-0,715$ & 0,074 & $0,680^{*}$ \\
\hline Item 9 & 0,511 & $-0,070$ & $-0,469 *$ & Item 34 & $-0,645$ & 0,153 & $0,530^{*}$ \\
\hline Item 10 & $-0,673$ & 0,079 & $0,631^{*}$ & Item 35 & $-0,560$ & 0,147 & $0,447^{*}$ \\
\hline Item 11 & 0,517 & $-0,259$ & $-0,293$ & Item 36 & $-0,737$ & 0,133 & $0,646^{*}$ \\
\hline Item 12 & $-0,605$ & 0,257 & 0,388 & Item 37 & $-0,685$ & 0,033 & $0,687^{*}$ \\
\hline Item 13 & $-0,762$ & $0,699 *$ & 0,128 & Item 38 & 0,583 & 0,004 & $-0,616^{*}$ \\
\hline Item 14 & $-0,636$ & $0,949 *$ & $-0,246$ & Item 39 & 0,462 & $-0,270$ & $-0,225$ \\
\hline Item 15 & $-0,668$ & 0,168 & $0,539 *$ & Item 40 & $-0,610$ & 0,059 & $0,584^{*}$ \\
\hline Item 16 & 0,547 & $-0,806^{*}$ & 0,202 & Item 41 & $-0,654$ & $0,786^{*}$ & $-0,070$ \\
\hline Item 17 & $-0,634$ & $-0,022$ & $0,645^{*}$ & Item 42 & $-0,790$ & $0,745^{*}$ & 0,113 \\
\hline Item 18 & $-0,697$ & 0,046 & $0,688^{*}$ & Item 43 & $-0,663$ & $0,635^{*}$ & 0,085 \\
\hline Item 19 & $-0,787$ & 0,512 & 0,334 & Item 44 & 0,565 & $-0,445^{*}$ & $-0,165$ \\
\hline Item 20 & $-0,602$ & $0,870^{*}$ & $-0,205$ & Item 45 & $-0,757$ & 0,158 & $0,643^{*}$ \\
\hline Item 21 & $-0,692$ & 0,209 & $0,526^{*}$ & Item 46 & $-0,624$ & $-0,319$ & 0,963 \\
\hline Item 22 & $-0,733$ & $0,664^{*}$ & 0,130 & Item 47 & $-0,527$ & 0,206 & 0,354 \\
\hline Item 23 & $-0,684$ & $0,807^{*}$ & $-0,059$ & Item 48 & $-0,642$ & $-0,229$ & $0,895^{*}$ \\
\hline Item 24 & $-0,729$ & $0,623^{*}$ & 0,166 & Item 49 & 0,444 & 0,089 & $-0,552 *$ \\
\hline Item 25 & $-0,690$ & 0,010 & $0,716^{*}$ & Item 50 & 0,505 & 0,105 & $-0,631 *$ \\
\hline
\end{tabular}

Nota. Resultados de duas análises fatoriais distintas, sendo uma unidimensional (coluna 2) e outra bidimensional (colunas 3 e 4); * Fator final de pertencimento do item.

$[F(4,686)=1,55 ; p=0,185)]$. Testes post-hoc de Bonferroni demonstraram as diferenças encontradas no fator IP são entre os níveis de escolaridade EMc e Dc, ESc e Dc, Ec e Mc, e Ec e Dc (Ver tabela 3).

Posteriormente, buscaram-se investigar diferenças nos níveis de ARC para sexo, religiosidade (presença e ausência) e tipo de instituição de ensino superior (IES; pública e privada), por meio do teste $t$ de Student. Foram encontradas diferenças significativas entre homens e mulheres tanto no fator IP $[t(630)=-5.36 ; p$ $\left.<0,01 ; \eta^{2}=0,41\right]$ quanto no fator CA $[t(689)=-3.40 ; p$ $\left.<0,01, \eta^{2}=0,31\right]$. Homens apresentaram escores estatisticamente superiores do que as mulheres nos fatores IP e CA [Ver Tabela 4]. O tamanho do efeito das diferenças foi moderado $(d=0,41)$ para o Fator IP e baixo para o fator CA $(d=0,31)$.

Também foram encontradas diferenças significativas entre religiosos e não religiosos tanto no fator IP $[t(664.64)=-8.00 ; p<0,001 ; d=0,61]$ quanto no fator CA $[t(684.17)=-10,44 ; \mathrm{p}<0,001 ; d=0,79]$. Pessoas 
Tabela 3.

ANOVA One-Way entre Escores da EARC - Iniciativa Pessoal e Niveis de Escolaridade

\begin{tabular}{|c|c|c|c|c|}
\hline Escolaridade & Média $(D P)$ & $\mathrm{D} M[99 \% \mathrm{IC}]$ & Sig. & Tamanho de efeito $(d)$ \\
\hline EMc & $67,17(10,79)$ & $0,98[-1,84-3,43]$ & 1,00 & - \\
\hline $\mathrm{ESc}$ & $66,19(10,22)$ & & & \\
\hline EMc & $67,17(10,79)$ & $2,74[-1,78-6,70]$ & 0,71 & - \\
\hline Ec & $64,44(10,48)$ & & & \\
\hline EMc & $67,17(10,79)$ & $-2,43[-6,39-1,09]$ & 1,00 & - \\
\hline $\mathrm{Mc}$ & $69,60(7.99)$ & & & \\
\hline EMc & $67,17(10,79)$ & $-6.04[-11,00--1,07]$ & 0,01 & 0,61 \\
\hline Dc & $73,21(6.04)$ & & & \\
\hline ESc & $66,19(10,22)$ & $1,76[-1,88-5,27]$ & 1,00 & - \\
\hline Ec & $64,44(10,48)$ & & & \\
\hline $\mathrm{ESc}$ & $66,19(10,22)$ & $-3.41[-6,80--0,51]$ & 0,18 & - \\
\hline Mc & 69,60 (7.99) & & & \\
\hline $\mathrm{ESc}$ & $66,19(10,22)$ & $-7.02[-9,55--4,23]$ & 0,00 & 0,71 \\
\hline Dc & $73.21(6.04)$ & & & \\
\hline Ec & $64.44(10,48)$ & $-5.16[-9,17--0,96]$ & $0,05^{*}$ & 0,55 \\
\hline $\mathrm{Mc}$ & $69.60(7.99)$ & & & \\
\hline Ec & $64.44(10,48)$ & $-8.78[-12,70--4,46]$ & 0,00 & 0,98 \\
\hline Dc & $73.21(6.04)$ & & & \\
\hline Mc & $69.60(7.99)$ & $-3.61[-7,15-0,27]$ & 0,78 & - \\
\hline Dc & $73.21(6.04)$ & & & \\
\hline
\end{tabular}

Nota. ${ }^{*}$ Diferenças estatisticamente significativas; $I P=$ Iniciativa Pessoal; $C A=$ crenças e afetos; $D P=$ desvio padrão; $\Delta M=$ diferença de média entre os grupos; $I C=$ Intervalo de Confiança; Sig = Significância estatística; $d=$ valor de $d$ de Cohen; EMc = Ensino Médio completo; ES $c=$ Ensino Superior completo; $E c=$ Especialização completa; $M c=$ Mestrado completo; $D c=$ Doutorado completo. Tamanho de efeitos não apresentados para resultados não significativos estatisticamente $(p>0,05)$.

que declararam não ter religião apresentaram escores estatisticamente superiores do que aqueles que afirmaram ter religião nos dois fatores (Ver tabela 4).

Também foi realizado o teste $t$ de Student para amostras independentes buscando verificar se, dentre os participantes que estavam ainda cursando o Ensino Superior $(n=262)$, havia diferenças nos níveis de atitude entre participantes que frequentaram universidade pública $(n$ $=178)$ ou privada $(n=84)$. Foram encontradas diferenças estatisticamente significativas no Fator IP $[t(260)=$ $3,12 ; p<0,004 ; d=0,41]$ com tamanho de efeito médio e não houve diferenças estatisticamente significativas em relação à CA $[t(260)=1,14 ; p=0,281]$. Os participantes que cursam o Ensino Superior em instituições públicas apresentaram escores mais altos do que os participantes de instituições privadas (Ver tabela 4).

Foi realizada uma ANOVA one-way para esses participantes que estão cursando o Ensino Superior, a fim de verificar diferenças nos escores de acordo com a área do curso dos indivíduos. Os cursos foram separados em três grupos: "Humanas" (incluindo Administração e Artes), "Exatas" e "Biológicas" (incluindo Química). Foram encontradas diferenças significativas no Fator $1[F(2,289)=6,3 ; p=0,002$; $d=0,42]$ e no Fator $2[F(2,289)=4.66 ; p=0,01 ; d$ $=0,31]$. Nos dois fatores os participantes das áreas de Exatas apresentaram escores maiores do que os participantes dos cursos de Humanas. Não houve diferenças estatisticamente significativas em relação aos cursos de Biológicas (ver Tabela 5).

Para verificar a relação entre os níveis de atitude em relação à ciência e a idade dos participantes, foram utilizadas correlações de Pearson. Foram encontradas correlações negativas muito fracas em ambos os fatores (Fator IP: $r=-0,10 ; p<0,01$; Fator CA: $r=-0,10$; $p<0,01)$. 
Tabela 4.

Testes t de Student para Avaliar Diferenças nos Niveis de ARC entre Sexo, Religião e Tipo de IES

\begin{tabular}{|c|c|c|c|c|c|c|}
\hline EARC & Sexo & Média $(D P)$ & $\mathrm{D} M[99 \% \mathrm{IC}]$ & $t$ & Sig & $\begin{array}{l}\text { Tamanho de } \\
\text { efeito }(d)\end{array}$ \\
\hline IP & $\begin{array}{l}\text { Feminino } \\
\text { Masculino }\end{array}$ & $\begin{array}{c}64,3(10,87) \\
68,85(9,1)\end{array}$ & $\begin{array}{c}-4.12[-6,2- \\
-2,05]\end{array}$ & $-5,36$ & $0,01 *$ & 0,41 \\
\hline CA & $\begin{array}{l}\text { Feminino } \\
\text { Masculino }\end{array}$ & $\begin{array}{c}109,00(13,5) \\
113,21(14,12)\end{array}$ & $\begin{array}{c}-4,22[-6,94- \\
-1,26]\end{array}$ & $-3,40$ & $0,00 *$ & 0,31 \\
\hline EARC & Religião & Média (DP) & $\mathrm{D} M[99 \% \mathrm{CI}]$ & t-valor & Sig & $\begin{array}{l}\text { Tamanho de } \\
\text { efeito (d) }\end{array}$ \\
\hline IP & $\begin{array}{l}\text { Sim } \\
\text { Não }\end{array}$ & $\begin{array}{c}64(10,7) \\
69,91(8,63)\end{array}$ & $\begin{array}{c}-5,91[-8,06- \\
-4,07]\end{array}$ & $-8,00$ & $0,001 *$ & 0,61 \\
\hline CA & $\begin{array}{l}\text { Sim } \\
\text { Não }\end{array}$ & $\begin{array}{l}106,14(13,66) \\
116,46(12,30)\end{array}$ & $\begin{array}{c}-10,32[-12,78- \\
-7,59]\end{array}$ & $-10,44$ & $0,001 *$ & 0,79 \\
\hline EARC & IES & Média (DP) & $\mathrm{DM}[99 \% \mathrm{CI}]$ & t-valor & Sig & $\begin{array}{l}\text { Tamanho de } \\
\text { efeito (d) }\end{array}$ \\
\hline IP & $\begin{array}{l}\text { Pública } \\
\text { Privada }\end{array}$ & $\begin{array}{c}67,14(9,66) \\
62,96(11,00)\end{array}$ & $\begin{array}{c}4,18[0,39- \\
7,80]\end{array}$ & 3,12 & $0,004 *$ & 0,41 \\
\hline CA & $\begin{array}{l}\text { Pública } \\
\text { Privada }\end{array}$ & $\begin{array}{l}111,31(14,79) \\
109,12(13,89)\end{array}$ & $\begin{array}{c}2,19[-3,55- \\
7,41]\end{array}$ & 1,14 & 0,281 & - \\
\hline
\end{tabular}

Nota. IP = Iniciativa Pessoal; $C A=$ Crenças e Afetos; IES = Instituição de Ensino Superior; $D P=$ desvio padrão; $\Delta M=$ Diferença de média entre os grupos; * diferenças estatisticamente significativas; Sig = Significância estatística; $d=$ valor de $d$ de Cohen. Tamanho de efeitos não apresentados para resultados não significativos estatisticamente $(p>0,05)$.

\section{Atitude em Relação à Ciência e Relações com Variáveis Psicológicas}

Também foram utilizadas correlações bivariadas de Pearson para verificar a relação entre os índices de ARC o construto Necessidade de conhecimento, mensurado pela SNC. Foram encontradas correlações positivas moderadas entre $\mathrm{SNC}$ e a EARC nos dois fatores (Fator IP: $r=0,55 ; p<0,01$; Fator CA: $r=0,42$; $p<0,01)$.

\section{Discussão}

Este estudo teve o objetivo de verificar evidências iniciais de validade da EARC. Em relação à estrutura fatorial, os resultados das análises indicaram uma maior plausibilidade para uma estrutura de dois fatores para a EARC (Iniciativa Pessoal, IP e Crenças e Afetos, $\mathrm{CA}$ ). $\mathrm{Na}$ estrutura da EARC, os itens de crenças e afetos em relação à ciência se agruparam em um fator (CA), enquanto os itens de característica mais comportamental, relacionados à disposição pessoal, compuseram outro fator (IP). A estrutura de três fatores proposta com base nos modelos clássicos de atitude (Rodrigues et al., 2001) não se mostrou como a mais adequada, conforme era esperado. Entretanto, a estrutura de dois fatores, que apresentou maior adequação psicométrica, é respaldada pela literatura científica, uma vez que existe um debate, dentro da Psicologia Social das atitudes, sobre cognição e afeto geralmente serem componentes coerentes entre si, mas nem sempre coerentes com aspectos comportamentais (Rodrigues et al., 2001). Isto é, as ações de uma pessoa nem sempre são guiadas de acordo com suas crenças e sentimentos, 
Tabela 5.

ANOVA One-Way para Avaliar Diferença de ARC entre as Areas das IES

\begin{tabular}{|c|c|c|c|c|c|}
\hline EARC & Área & Média $(D P)$ & $\mathrm{D} M[99 \% \mathrm{CI}]$ & Sig & $\begin{array}{c}\text { Tamanho de } \\
\text { efeito }(d)\end{array}$ \\
\hline \multirow[t]{3}{*}{ IP } & $\begin{array}{c}\text { Humanas } \\
\text { Exatas }\end{array}$ & $\begin{array}{l}65,82(9,85) \\
70,43(8,93)\end{array}$ & $\begin{array}{c}-4,61[-7,91- \\
-1,28]\end{array}$ & $0,030^{*}$ & 0,48 \\
\hline & $\begin{array}{l}\text { Humanas } \\
\text { Biológicas }\end{array}$ & $\begin{array}{c}65,82(9,85) \\
68,94(10,12)\end{array}$ & $-3,11[-7,15-0,97]$ & 0,091 & - \\
\hline & $\begin{array}{c}\text { Exatas } \\
\text { Biológicas }\end{array}$ & $\begin{array}{c}70,43(8,93) \\
68,94(10,12)\end{array}$ & $1,49[-2,74-6,07]$ & 1,000 & - \\
\hline \multirow[t]{3}{*}{ CA } & $\begin{array}{c}\text { Humanas } \\
\text { Exatas }\end{array}$ & $\begin{array}{l}109,39(13,37) \\
115,11(12,48)\end{array}$ & $\begin{array}{c}-5,72[-10,35- \\
-0,93]\end{array}$ & $0,007 *$ & 0,44 \\
\hline & $\begin{array}{l}\text { Humanas } \\
\text { Biológicas }\end{array}$ & $\begin{array}{l}109,39(13,37) \\
111,23(13,23)\end{array}$ & $-1,84[-6,96-3,91]$ & 1,000 & - \\
\hline & $\begin{array}{c}\text { Exatas } \\
\text { Biológicas } \\
\end{array}$ & $\begin{array}{l}115,11(12,48) \\
111,23(13,23)\end{array}$ & $3,88[-2,00-9,37]$ & 0,255 & - \\
\hline
\end{tabular}

Nota. ${ }^{*}$ Diferenças estatisticamente significativas; $I P=$ Iniciativa Pessoal; $C A=$ Crenças e Afetos. Tamanho de efeitos não apresentados para resultados não significativos estatisticamente $(p>0,05)$.

o que talvez explique a dissonância gerada entre esses e o componente comportamental das atitudes (Bienemann \& Damásio, 2017). Desse modo, crenças e afetos favoráveis não necessariamente se expressariam em comportamentos favoráveis e vice-versa (Kruglanski et al., 2015).

No que diz respeito às associações da EARC com outras variáveis, a hipótese de que ARC se correlacionaria positivamente com grau de escolaridade foi parcialmente confirmada, uma vez que somente o fator IP se correlacionou. Entretanto, esse resultado faz sentido, uma vez que se espera ocorrer um aumento da familiaridade com a ciência ao longo da vida acadêmica, em especial na pós-graduação (Antonioli, 2012; Perry et al., 2012). Por isso, os níveis de pós-graduação acabam atuando como filtro por, naturalmente, já concentrarem indivíduos dispostos a fazer pesquisa científica - o que ficou evidenciado por meio da correlação exclusiva com o fator IP, referente à disposição para um fazer científico.

Em relação ao sexo dos participantes, a hipótese de que homens teriam ARC mais favorável do que as mulheres se confirmou, expressa em uma diferença de tamanho de efeito moderado para o fator IP, e baixo para o fator CA. Esse resultado está em consonância com o fato de que homens tendem a se envolver mais com ciência do que mulheres, especialmente devido a questões culturais e sociais (Christidou, 2011; Smith et al., 2014). Além disso, estereótipos em relação às mulheres na ciência estão estritamente relacionados com o engajamento destas na área (Miller, Eagly, \& Linn, 2015), sendo que muitas crianças já acabam assimilando, em poucos anos de idade, que inteligência e brilhantismo são características masculinas (Bian, Leslie, \& Cimpian, 2017) - o que tende a restringir a gama de carreiras escolhidas pelas mulheres no futuro (Cadaret, Hartung, Subich, \& Weigold, 2017; Carli, Alawa, Lee, Zhao, \& Kim, 2016; Stout, Grunberg, \& Ito, 2016; Upson \& Friedman, 2012).

Além disso, verificou-se as associações entre ARC e necessidade de conhecimento, mensurada pela SNC. Foram encontradas correlações moderadas entre EARC e SNC, o que parece estar de acordo com a literatura. $\mathrm{O}$ construto mensurado pela $\mathrm{SNC}$ se refere ao quanto o sujeito valoriza e busca levantar conhecimentos de diversas áreas ao longo da vida (Schnell, 2009), o que parece compatível com a atividade científica e com as características pessoais dos cientistas (Feist, 2012). As correlações moderadas indicam, portanto, que os construtos são diferentes, porém se associam entre si.

Outras relações entre variáveis foram verificadas em caráter exploratório, uma vez que tais verificações não estavam previstas nas hipóteses aventadas inicialmente. Por exemplo, foi observado que religiosos apresentaram ARC menos favorável em ambos os fatores da EARC. Tal fato pode estar relacionado à 
natureza da crença religiosa, baseada em dogmatismo em vez de racionalismo, e ao próprio conflito histórico existente entre ciência e religião (Pennycook, Cheyne, Barr, Koehler, \& Fugelsang, 2014). No mais, níveis altos de religiosidade parecem contribuir tanto com um ceticismo em relação à ciência, quanto a uma menor disposição de se engajar em práticas científicas (Rutjens, Sutton, \& van der Lee, 2018).

O tipo de instituição de Ensino Superior (IES) dos participantes também apresentou uma relação significativa sobre a ARC. Os estudantes de IES públicas tiveram ARC mais favorável do que os que estudavam em IES particulares, possivelmente devido a uma maior tradição com programas de pós-graduação nas IES públicas. Como este é o primeiro estudo a comparar ARC entre estudantes de universidades públicas e particulares, não há referências similares e as conclusões tendem a ficar no campo das hipóteses. Entretanto, em um estudo similar realizado anteriormente sobre ARC especificamente na área da Psicologia (Bienemann \& Damásio, no prelo), foi encontrado um resultado oposto - isto é, estudantes de Psicologia de IES privadas demonstraram níveis mais positivos de ARC do que estudantes de IES públicas. Tal dado pode indicar uma particularidade do curso de Psicologia, e talvez mais genericamente na área de Humanas, o que fora evidenciado pelos resultados encontrados no presente estudo - em que os estudantes dos cursos de Exatas tiveram níveis maiores de ARC do que os de Humanas. Em relação a este fato, sabe-se que em alguns cursos de humanas no Brasil, como o de Psicologia, existe ênfase significativa em intervenção e discussões políticas, o que também inclui críticas às pesquisas empíricas (Gouveia, 2015), fator que poderia levar a atitude menos favorável à ciência e, consequentemente, a menor iniciativa pessoal para buscar metas relacionadas à ciência. Além disso, no Brasil, as áreas de Humanas possuem significativa atuação por parte de correntes políticas e filosóficas com visões epistemologicamente pós-modernistas (Castañon, 2009a), o que também pode se constituir como um entrave ideário para uma maior aceitação da prática científica nessas áreas.

\section{Considerações Finais}

Em geral, os estudos de ARC são voltados para um público específico (infantil ou escolar, por exemplo) ou para uma determinada área do saber (e.g., Biologia ou Psicologia). A EARC é uma escala que pode ser utilizada para diferentes amostras e avalia um nível de atitude em relação à ciência em geral, sem especificidade de área. Os resultados deste e de futuros estudos também podem ser importantes para se compreender os impactos pessoais e sociais que diferentes níveis de ARC apresentam em determinados contextos e instituições, uma vez que foram testadas relações entre grupos que não haviam sido analisadas anteriormente nos estudos sobre ARC.

Não foi possível identificar outros fatores que talvez influenciem os níveis de ARC dos participantes, tais como conhecimento sobre ciência e entendimento sobre a natureza da ciência e sobre o método científico (Allum, Sibley, Sturgis, \& Stoneman, 2014; Chen et al., 2013) e isso se constitui como uma limitação do estudo. Outra limitação foi não ter investigado como se dá a variação da ARC entre os estudantes de graduação, além das áreas em que os cursos estão inseridos (Exatas e Humanas, por exemplo). No mais, variáveis acadêmicas externas, tais como medidas de coeficiente de rendimento (CR), não foram utilizadas neste estudo, mas talvez possam funcionar como indicativos importantes dos níveis de ARC entre os estudantes. No contexto atual, faz-se extremamente importante o incentivo ao pensamento científico entre diferentes segmentos da população. Nesse sentido, espera-se que sejam feitos outros estudos que busquem ampliar o entendimento sobre ARC, seus preditores e o impacto dessas atitudes entre diversos setores sociais e institucionais.

\section{Referências}

Allum, N., Sibley, E., Sturgis, P., \& Stoneman, P. (2014). Religious beliefs, knowledge about science and attitudes towards medical genetics. Public Understanding of Science, 23(7), 833-849 doi:0963662513492485

Antonioli, P. M. (2012). Atitudes, valores e crenças de alunos do ensino médio em relação à ciência e a tecnologia (Dissertação de mestrado). Centro Federal de Educação Tecnológica Celso Suckow da Fonseca. Rio de Janeiro, RJ, Brasil. Recuperado de http://dippg.cefet-rj. $\mathrm{br} /$ index.php?option $=$ com_docman\&task $=$ doc download\&gid $=968 \&$ Itemid $=166$

Bian, L., Leslie, S. J., \& Cimpian, A. (2017). Gender stereotypes about intellectual ability emerge early and influence children's interests. Science, 355(6323), 389-391. doi: 10.1126/science.aah6524

Bienemann, B., \& Damásio, B. F. (no prelo). Atitudes em relação à ciência na Psicologia: Relações com características sociodemográficas entre estudantes 
e profissionais brasileiros. Temas em Psicologia, 27(2), 2019.

Bienemann, B., \& Damásio, B. F. (2017). Desenvolvimento e validação de uma escala de atitude em relação à ciência na psicologia. Avaliação Psicológica, 16(4), 489-497. doi: 10.15689/ap.2017.1604.13409

Blalock, C. L., Lichtenstein, M. J., Owen, S., Pruski, L., Marshall, C., \& Toepperwein, M. (2008). In pursuit of validity: A comprehensive review of science attitude instruments 1935-2005. International Journal of Science Education, 30(7), 961-977. doi:10.1080/09500690701344578

Bland, S. (2014). Incommensurability, relativism, and the epistemic authority of science. Episteme, 11(04), 463-473. doi:10.1017/epi.2014.2

Borsa,J.C.,Damásio,B.F.,\&Bandeira,D. R. (2012).Adaptação e validação de instrumentos psicológicos entre culturas: algumas considerações. Paidéia, 22(53), 423-432. doi:10.1590/1982-43272253201314

Brown, T. A. (2006). Confirmatory factor analysis for applied research. New York: The Guilford Press. Recuperado de http://books.google.com/books

Cadaret, M. C., Hartung, P. J., Subich, L. M., \& Weigold, I. K. (2017). Stereotype threat as a barrier to women entering engineering careers. Journal of Vocational Behavior, 99, 40-51. doi: 10.1016/j.jvb.2016.12.002

Carli, L. L., Alawa, L., Lee, Y., Zhao, B., \& Kim, E. (2016). Stereotypes about gender and science: Women $\neq$ scientists. Psychology of Women Quarterly, 40(2), 244-260. doi: 10.1177/0361684315622645

Castañon, G. A. (2009a). Science Wars: Uma guerrilha contra a ciência moderna. Boletim Interfaces da Psicologia, 2(1), 70-76. Recuperado de http://ufrrj. br/seminariopsi/2009/boletim2009-1/boletim. pdf\#page $=71$

Castañon, G. A. (2009b). Psicologia como ciência moderna: Vetos históricos e status atual. Temas em Psicologia, 17(1), 21-36. Recuperado de http:/ / pepsic.bvsalud. org/scielo.php?script $=$ sci_arttext\&pid $=$ S1413$-389 \times 2009000100004 \& \operatorname{lng}=p t \& \operatorname{lng}=$

Chen, S., Chang, W. H., Lieu, S. C., Kao, H. L., Huang, M. T., \& Lin, S. F. (2013). Development of an empirically based questionnaire to investigate young students' ideas about nature of science. Journal of Research in Science Teaching, 50(4), 408-430. doi:10.1002/tea.21079
Christidou, V. (2011). Interest, attitudes and images related to science: Combining students' voices with the voices of school science, teachers, and popular science. International Journal of Environmental \& Science Education, 6(2), 141-159. Recuperado de http://eric.ed.gov/?id=EJ944846

Cohen, J. (1988). Statistical powver analysis for the behavioral sciences (2nd ed.). New Jersey: Lawrence Erlbaum.

Constantino, P. M. B. (2004). Aquisição do conbecimento $e$ atitudes científicas na infância (Dissertação de mestrado). Instituto Superior de Psicologia Aplicada, Lisboa, Portugal. Recuperado de http://repositorio.ispa.pt/handle/10400.12/440

Cunha, A. M. (2008). Ciência, Tecnologia e Sociedade na Óptica Docente: Construção e Validação de Uma Escala de Atitudes (Dissertação de mestrado). Universidade Estadual de Campinas. Campinas, SP, Brasil. Recuperado de http://repositorio.unicamp.br/handle/ REPOSIP/252114

Damásio, B. F., Koller, S. H., \& Schnell, T. (2013). Sources of Meaning and Meaning in Life Questionnaire (SoMe): Psychometric properties and sociodemographic findings in a large Brazilian sample. Acta de Investigación Psicológica, 3(3), 12051227. doi:10.1016/S2007-4719(13)70961-X

Feist, G. J. (2012). Predicting interest in and attitudes toward science from personality and need for cognition. Personality and Individual Differences, 52(7), 771-775. doi: 10.1016/j.paid.2012.01.005

Germann, P. J. (1988). Development of the attitude toward science in school assessment and its use to investigate the relationship between science achievement and attitude toward science in school. Journal of Research in Science Teaching, 25(8), 689-703. doi: 10.1002/tea.3660250807

Gouveia, V. V. (2015). Psicologia Social como ciência e prática: O que pensam pesquisadores brasileiros? Psicologia Teoria e Pesquisa, 31(4), 491-500. doi:10.1590/0102-37722015042306491500

Greenwald, A. G., Brock, T. C., \& Ostrom, T. M. (Eds.). (2013). Psychological foundations of attitudes. Academic Press. Recuperado de de https://books.google. com.br/books

Haukoos, J. S., \& Lewis, R. J. (2005). Advanced statistics: Bootstrapping confidence intervals for statistics with "difficult" distributions. Academic 
Emergency Medicine, 12(4), 360-365. doi: 10.1197/j. aem.2004.11.018

Horn, J. L. (1965). A rationale and technique for estimating the number of factors in factor analysis. Psychometrika, 30(1), 179-185. doi:10.1007/ BF02289447

Krapp, A., \& Prenzel, M. (2011). Research on interest in science: Theories, methods, and findings. International Journal of Science Education, 33(1), 27-50. doi: 10.1080/09500693.2010.518645

Kruglanski, A. W., Jasko, K., Chernikova, M., Milyavsky, M., Babush, M., Baldner, C., \& Pierro, A. (2015). The rocky road from attitudes to behaviors: Charting the goal systemic course of actions. Psychological Review, 122(4), 598-620. doi:10.1037/a0039541

Kuhn, D. (2005). Education for thinking. Harvard University Press.

Lilienfeld, S. O. (2010). Can psychology become a science? Personality and Individual Differences, 49(4), 281-288. doi:10.1016/j.paid.2010.01.024

Lobato, E. \& Zimmerman, C. (2018). The Psychology of (Pseudo) Science: Cognitive, Social, and Cultural Factors. 21-42. Em Hecht, D. K., Lobato, E., Zimmerman, C., Blanco, F., Matute, H., Simonton, D. K., ... \& Ball, D. (Eds). Pseudoscience: The Conspiracy Against Science. MIT Press.

Lorenzo-Seva, U., \& Ferrando, P. J. (2006). Factor (9.2) [Computer program]. Recuperado de http://psico. fcep.urv.es/utilitats/factor/

Lorenzo-Seva, U., Timmerman, M. E., \& Kiers, H. A. (2011). The hull method for selecting the number of common factors. Multivariate Behavioral Research, 46(2), 340-364. doi:10.1080/00273171.2011.56452 7

Miller, D. I., Eagly, A. H., \& Linn, M. C. (2015). Women's representation in science predicts national gender-science stereotypes: Evidence from 66 nations. Journal of Educational Psychology, 107(3), 631. doi: $10.1037 / \mathrm{edu} 0000005$

Muthén, L. K., \& Muthén, B. O. (2012). Mplus: Statistical analysis with latent variables. User's guide. Los Angeles: Muthén \& Muthén. Recuperado de https://www. statmodel.com/download/usersguide/MplusUserGuideVer_7.pdf
Osborne, J., Simon, S., \& Collins, S. (2003). Attitudes towards science: A review of the literature and its implications. International Journal of Scientific Education, 25(9), 1049-1079. doi:10.1080/095006903200 0032199

Pardo, R., \& Calvo, F. (2016). Attitudes toward science among the European public: A methodological analysis. Public Understanding of Science, 11(2), 155195. doi:10.1088/0963-6625/11/2/305

Pennycook, G., Cheyne, J. A., Barr, N., Koehler, D. J., \& Fugelsang, J. A. (2014). Cognitive style and religiosity: The role of conflict detection. Memory \& Cognition, 42(1), 1-10. doi: 10.3758/ s13421-013-0340-7

Perry, B. L., Link, T., Boelter, C., \& Leukefeld, C. (2012). Blinded to science: Gender differences in the effects of race, ethnicity, and socioeconomic status on academic and science attitudes among sixth graders. Gender and Education, 24(7), 725-743. doi:10.1080/09540253.2012.685702

Pituch, K. A., \& Stevens, J. P. (2015). Applied multivariate statistics for the social sciences: Analyses with $S A S$ and IBM's SPSS. Routledge.

Popper, K. R. (2004). A lógica da pesquisa científica. (Hegenberg, L. \& Mota, O. S., Trad.). São Paulo: Editora Cultrix. (Obra original publicada em 1959).

Potvin, P., \& Hasni, A. (2014). Interest, motivation and attitude towards science and technology at K-12 levels: A systematic review of 12 years of educational research. Studies in Science Education, 50(1), 85-129. doi: 10.1080/03057267.2014.881626

Reuben, E., Sapienza, P., \& Zingales, L. (2014). How stereotypes impair women's careers in science. Proceedings of the National Academy of Sciences, 111(12), 4403-4408. doi: 10.1073/pnas.1314788111

Rodrigues, A., Assmar, E. M. L., \& Jabonski, B. (2001). Psicologia Social. [23 ${ }^{\mathrm{a}}$ ed.]. Petrópolis: Vozes.

Rutjens, B. T., Sutton, R. M., \& van der Lee, R. (2018). Not all skepticism is equal: Exploring the ideological antecedents of science acceptance and rejection. Personality and Social Psychology Bulletin, 44(3), 384-405. doi: 10.1177/0146167217741314

Sagan, C. (2006). O mundo assombrado pelos demônios: A ciência vista como uma vela no escuro. (Eichemberg, R., Trad.). São Paulo: Companhia das letras. (Original publicado em 1995). 
Schnell, T. (2009). The Sources of Meaning and Meaning in Life Questionnaire (SoMe): Relations to demographics and well-being. The Journal of Positive Psychology, 4(6), 483-499. doi: 10.1080/17439760903271074

Schnell, T., \& Becker, P. (2007). Der Fragebogen zи Lebensbedeutungen und Lebenssinn (LeBe) [The Sources of Meaning and Meaning in Life questionnaires]. Göttingen, Germany: Hogrefe.

Smith, T. J., Pasero, S. L., \& McKenna, C. M. (2014). Gender effects on student attitude toward science. Bulletin of Science, Technology \& Society, 34(1-2), 7-12. doi:10.1177/0270467614542806

Stout, J. G., Grunberg, V. A., \& Ito, T. A. (2016). Gender roles and stereotypes about science careers help explain women and men's science pursuits. Sex Roles, 75(9-10), 490-499. doi: 10.1007/ s11199-016-0647-5

Timmerman, M. E., \& Lorenzo-Seva, U. (2011). Dimensionality assessment of ordered polytomous items with parallel analysis. Psychological Methods, 16(2), 209-220. doi:10.1037/a0023353
Upson, S., \& Friedman, L. F. (2012). Where are all the female geniuses? Scientific American Mind, 23(5), 6365. doi: 10.1038/scientificamericanmind1112-63

van Aalderen-Smeets, S. I., \& van der Molen, J. H. W. (2015). Improving primary teachers' attitudes toward science by attitude-focused professional development. Journal of Research in Science Teaching, 52(5), 710-734. doi:10.1002/tea.21218

Wang, T. L., \& Berlin, D. (2010). Construction and validation of an instrument to measure Taiwanese elementary students' attitudes toward their science class. International Journal of Science Education, 32(18), 2413-2428. doi:10.1080/09500690903431561

Weinburgh, M. (1995). Gender differences in student attitudes toward science: A meta-analysis of the literature from 1970 to 1991. Journal of Research in Science Teaching, 32(4), 387-398. doi: 10.1002/ tea.3660320407

Recebido em: 20/06/2016

Reformulado em: 07/12/2018

Aprovado em: 12/12/2018 
Sobre os autores:

Felipe Carvalho Novaes é Mestre em Psicologia Clínica pela Pontifícia Universidade Católica do Rio de Janeiro. ORCID: 0000-0002-4534-5059

Bheatrix Bienemann é psicóloga formada pela Universidade Federal do Rio de Janeiro e mestra em Psicologia Clínica pela Pontifícia Universidade Católica do Rio de Janeiro.

ORCID: 0000-0003-4291-4612

Fernanda de Oliveira Paveltchuk é psicóloga formada pela Universidade Federal do Rio de Janeiro (UFRJ) e mestra em Psicologia Clínica pela Pontifícia Universidade Católica do Rio de Janeiro (PUC-Rio).

ORCID: 0000-0002-8319-874X

Pedro Henrique Teodoro Siqueira é psicólogo formado pela Universidade Federal do Rio de Janeiro.

ORCID: 0000-0001-8118-2632

Bruno Figueiredo Damásio é professor do Instituto de Psicologia da Universidade Federal do Rio de Janeiro (UFRJ), chefe do Departamento de Psicometria e coordenador do Laboratório de Psicometria e Psicologia Positiva (LP3).

ORCID: 0000-0002-1150-092X

E-mail: bf.damasio@gmail.com

Contato com os autores:

Rua Marquês de São Vicente, 225, Gávea

Rio de Janeiro-RJ, Brasil

CEP: 22451-900 